\title{
Investigation of plastic deformation of aluminum alloys using wavelet transforms of acoustic emission signals
}

\author{
A. A. Dmitriev ${ }^{1}$, V. V. Polyakov ${ }^{1,2, \dagger}$ A. A Lependin ${ }^{1}$ \\ †pvv@asu.ru \\ ${ }^{1}$ Altai State University, 16, Lenina pr., Barnaul, 656049, Russia \\ ${ }^{2}$ Institute of Strength Physics and Materials Science SB RAS, 2/4, pr. Akademicheskii, Tomsk, 634055, Russia
}

\begin{abstract}
On the example of aluminum alloy AMg5 м plastic deformation and fracture under static loading was investigated. For the material being loaded, a stage of linear hardening, two stages of parabolic hardening and a fracture stage were identified. A change in the form of acoustic emission signals was established with a change in the region of strain hardening. The plastic yielding in the first region was accompanied by the formation of an acoustic emission peak, which was replaced in the next stage by high-amplitude oscillations. Further, for the region of discontinuous yielding, bursts of signals of different amplitude were observed, reflecting the dynamics of the formation of localized deformation bands. The change in the acoustic emission signals reflected the evolution of the deformation hardening processes. To describe the effect of the stagedness of deformation processes on the parameters of acoustic emission, the initial acoustic-emission signal was divided into separate time blocks. To each of these blocks, a discrete wavelet transform was applied. It characterized the time dependence of the waveform on the specific section of the strain hardening curve corresponding for each block. The obtained wavelet decomposition coefficients were processed using principal component analysis. They were plotted on the plane of the first principal components. The points of the multidimensional space corresponded to different regions were divided into partially overlapping clusters. The results of the work showed that the wavelet decomposition coefficients of acoustic emission signal could be used for diagnostics of the stages of strain hardening in aluminum alloys.
\end{abstract}

Keywords: acoustic emission, discrete wavelet transformation, principal component analysis.

УДК 539.42

\section{Исследование пластической деформации алюминиевых}

\section{сплавов с использованием вейвлет-преобразований сигналов акустической эмиссии}

\author{
Дмитриев А. А. ${ }^{1}$, Поляков В. В. ${ }^{1,2, \dagger}$, Лепендин А. А. ${ }^{1}$ \\ ${ }^{1}$ Алтайский государственный университет, пр. Ленина, 61, Барнаул, 656049, Россия \\ ${ }^{2}$ Институт физики прочности и материаловедения СО РАН, пр. Академический, 2/4, Томск, 634055, Россия
}

\begin{abstract}
Исследована акустическая эмиссия в процессе развития пластической деформации в алюминий-магниевых сплавах АМг5м при статическом растяжении. Для нагружаемого материала были выявлены стадия линейного упрочнения, две стадии параболического упрочнения и стадия предразрушения. Установлено изменение вида акустикоэмиссионных сигналов при смене стадий деформационного упрочнения. Пластическое течение на первой стадии сопровождалось формированием пика акустической эмиссии, который сменялся на следующей стадии высокоамплитудными осцилляциями. Далее для стадии прерывистой текучести наблюдались всплески сигналов различной амплитуды, отражавшие динамику формирования и развития полос локализованной деформации. Для описания влияния стадийности деформационных процессов на параметры акустической эмиссии исходный акустико-эмиссионный сигнал разбивался на отдельные временные блоки. К каждому из таких блоков применялось дискретное вейвлет-преобразование, характеризовавшее временную зависимость формы сигналов на конкретном участке кривой деформационного упрочнения. Примененная вейвлет-обработка обеспечила выделение огибающей для низкочастотных компонент временных блоков регистрируемых акустико-эмиссионных сигналов. Полученные коэффициенты вейвлет-разложений обрабатывались с помощью проекционных методов многомерного анализа данных и анализировались на плоскости первых главных компонент. Установлено, что точки многомерного пространства, характеризовавшие блоки сигналов на отдельных стадиях, разделялись на частично перекрывавшиеся кластеры, соответствовавшие этим стадиям. Результаты работы показывают, что коэффициенты вейвлет-разложений акустико-эмиссионных сигналов могут быть использованы в качестве релевантных параметров при исследовании стадий деформационного упрочнения алюминий-магниевых сплавов.
\end{abstract}

Ключевые слова: акустическая эмиссия, дискретное вейвлет преобразование, метод главных компонент. 


\section{1. Введение}

Процессы пластической деформации и разрушения, протекающие в материале при нагружении, проявляются в виде акустической эмиссии. Регистрируемые при акустической эмиссии сигналы отражают одновременное действие различных физических механизмов, определяющих особенности деформационных процессов на различных стадиях нагружения. Существующие методы исследования сигналов акустической эмиссии обычно основываются на анализе числа импульсов и их частотного спектра, на основе этих характеристик делаются выводы об источниках излучения и конкретных механизмах деформации [1]. В то же время в силу сложности формирующихся сигналов непосредственная идентификация протекающих процессов весьма затруднительна, что накладывает ограничения на применение метода акустической эмиссии в задачах диагностики материалов. Это обстоятельство привело к появлению более сложных методов анализа акустической эмиссии, связанных с привлечением новых информативных характеристик $[2,3]$. В настоящей работе в качестве признаков, характеризующих сигналы акустической эмиссии, предложено использовать коэффициенты многоуровневых дискретных вейвлет-преобразований [4-6], описывающие энергетические и частотные особенности сигналов, с последующей кластеризацией получаемых данных на основе метода главных компонент $[7,8]$.

\section{2. Экспериментальные результаты}

В качестве материала для испытаний использовался алюминиевый сплав АМг5м, структура и деформационное поведение которого изучались достаточно подробно [9 - 11]. Образцы имели стандартную плоскую форму для испытаний на статическое одноосное растяжение с размерами рабочей части $5 \times 15 \times 50$ мм. Нагружение проводилось по «жесткой» схеме с постоянной скоростью растяжения. При измерениях регистрировались приложенная нагрузка и абсолютное удлинение, по которым определялись истинное напряжение $\sigma$ и истинная (логарифмическая) деформация $\varepsilon$. Для выделения стадий деформационного упрочнения рассчитывался в истинных координатах коэффициент деформационного упрочнения $K$ [12]. Полученные данные приведены на Рис. 1а в виде деформационных кривых $\sigma(\varepsilon)$ и зависимостей $K(\varepsilon)$.

Как видно из Рис. 1a, после перехода к пластическому течению для процесса нагружения можно выделить несколько стадий: стадию линейного упрочнения I, две стадии параболического упрочнения II и III, различавшиеся по поведению коэффициента деформационного упрочнения, и стадию предразрушения IV. Первые две стадии связаны с равномерной пластической деформацией, на третьей стадии возникает неустойчивость пластического течения, проявляющаяся в виде явления прерывистой текучести с локализацией деформации в полосах Портевена - Ле Шателье $[13,14]$.

Одновременно с механическими характеристиками по методике [15] регистрировались сигналы акустиче- ской эмиссии, измерявшиеся в частотном интервале от 50 до 700 кГц. Эти сигналы представлены на Рис. $1 \mathrm{~b}$ в виде зависимости среднеквадратичных значений напряжения $U_{e}$, записанных с частотой дискретизации 2,5 МГц, от деформации $\varepsilon$ (для удобства анализа использовалось отношение $U_{e} / U_{n}$, где $U_{n}-$ значение шума в ненагруженном состоянии).

Рис. $1 \mathrm{~b}$ свидетельствует о существенном различии вида сигналов акустической эмиссии для отдельных стадий. Пластическое течение сопровождалось формированием пика акустической эмиссии на стадии I, сменявшимся высокоамплитудными осцилляциями для стадии II и далее, в области прерывистой текучести (стадия III) - всплесками сигналов различной амплитуды. Пик акустической эмиссии может быть связан с выходом на границы раздела дислокационных потоков в условиях монотонного накопления деформации. Участок осциллирующей акустической эмиссии, согласно [11], связывается с началом формирования полос локализованной деформации. Всплески акустико-эмиссионных сигналов на третьей стадии отражают скачки упругого напряжения, обусловленные динамикой формирования и развития полос [16]. С позиций мезоскопического описания акустическое излучение для этой стадии связано с одновременным протеканием процессов равномерной деформации и ее локализацией вследствие явления прерывистой текучести [17]. Отличительные особенности акустической эмиссии на стадии

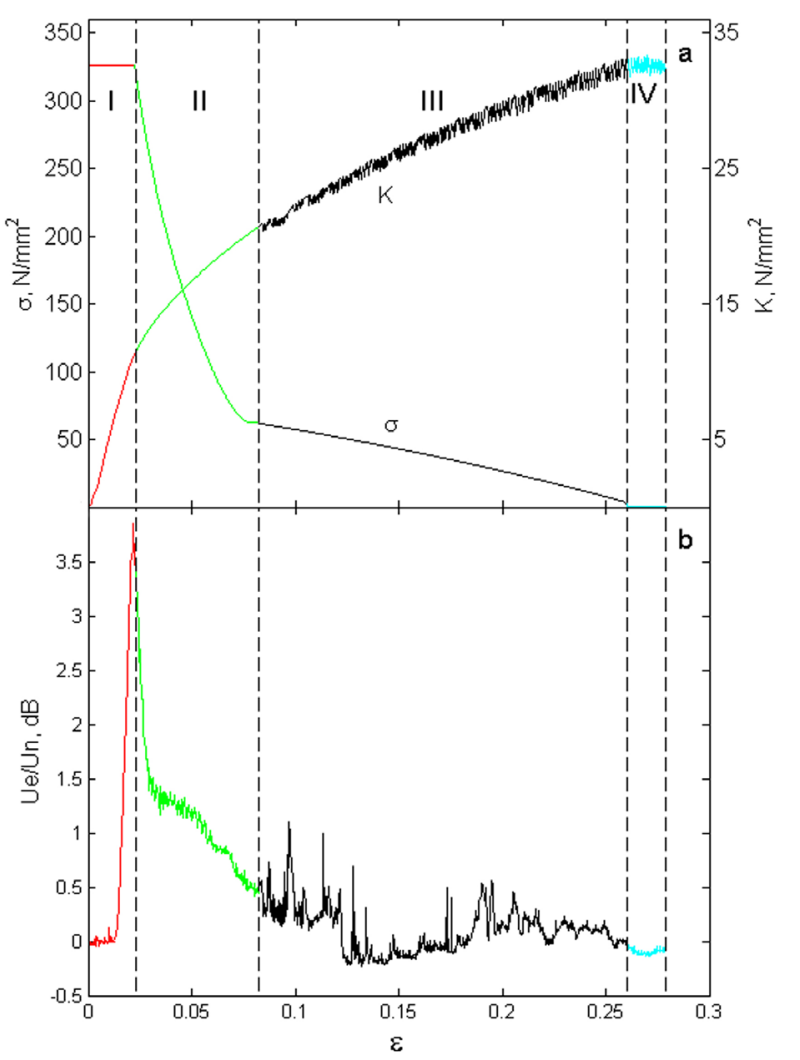

Рис. 1. Деформационное упрочнение (а) и акустическая эмиссия (b) для алюминий-магниевого сплава. I, II, III, IV - стадии пластической деформации.

Fig. 1. Strain hardening curve (a) and acoustic emission signal (b) of aluminum-magnesium alloy. I, II, III, IV are regions of plastic deformation. 
предразрушения IV, по-видимому, могут быть связаны с дополнительным вкладом от зарождения микротрещин, приводящим к последующему разрушению образца [10]. Для анализа акустико-эмиссионных сигналов на разных стадиях могут быть также привлечены представления о различных типах автоволнового пластического течения на этих стадиях [18] и другие макроскопические интерпретации.

\section{3. Применение вейвлет-разложений сигналов акустической эмиссии}

Результаты Рис. 1 показывают, что вид сигналов акустической эмиссии отражает протекавшие в материале деформационные процессы. В то же время при практической диагностике материалов часто используется сигнал малой длительности, описывающий текущее нагруженное состояние. Использовать подобный сигнал для диагностики стадии упрочнения и действующих на данной стадии процессов достаточно затруднительно. Этим определяется смысл применения к измеряемым сигналам вейвлет-преобразования, которое характеризует форму акустико-эмиссионных сигналов для выделяемого временного интервала и соответствующего участка кривой деформационного упрочнения.

При измерениях весь регистрировавшийся сигнал разбивался на временные блоки длительностью $10^{7}$ дискретных отсчетов при общем числе отсчетов за весь период измерения $\sim 10^{9}$. Отдельный блок характеризовал поведение материала на соответствующем участке кривой $\sigma(\varepsilon)$. Далее к каждому из этих блоков применялось многоуровневое дискретное вейвлет-преобразование [4, 19]. В основе этого преобразования лежит одновременная фильтрация сигнала низкочастотным и высокочастотным фильтрами, построенными на основе заданных вейвлет-функций:

$$
y_{l}[n]=\sum_{k=-\infty}^{\infty} \mathrm{X}[k] g[2 n-k], \quad y_{h}[n]=\sum_{k=-\infty}^{\infty} \mathrm{X}[k] h[2 n-k],
$$

где $y_{l}$ и $y_{h}-$ коэффициенты вейвлет-разложения для низкочастотного и высокочастотного фильтров соответственно, $g$ и $h-$ значения коэффициентов этих фильтров, X - обрабатываемый дискретный сигнал, $k-$ индекс, определяющий дискретное значение сигнала. Для обработки применялось 9-уровневое вейвлет-разложение по базису вейвлет-функций Добеши, уровень разложения определялся по минимальной погрешности, вносимой численными расчетами [4]. Далее полученные коэффициенты обрабатывались с помощью преобразования Гильберта [20], в котором для коэффициентов $y_{l}[n]$ строились значения $y_{g}[n]$ с исходной амплитудой и фазой, смещенной на $\pi / 2$. В качестве новых информативных характеристик акустической эмиссии использовались коэффициенты равные значениям мгновенной амплитуды и описывавшие огибающую для конкретного блока исходных сигналов:

$$
E[n]=\sqrt{y_{l}[n]^{2}+y_{g}[n]^{2}} .
$$

В целом применяемая вейвлет-обработка обеспечивала выделение огибающей для низкочастотных компо- нент временных блоков регистрируемых сигналов с одновременной фильтрацией шумов в этих блоках.

Для выявления связи коэффициентов $E[n]$ со стадиями деформационного упрочнения был привлечен метод главных компонент $[7,8]$. Согласно этому методу совокупность информативных характеристик, описывавших выделенный блок сигналов, рассматривалась как точка многомерного пространства. Группы точек, описывавших блоки с близкими свойствами, располагались в многомерном пространстве вблизи друг от друга и образовывали кластеры. Результаты расчетов, представленные в виде проекций многомерных данных на плоскость первых главных компонент РС1 и РС2, приведены на Рис. 2.

Как следует из Рис. 2, точки I - IV, описывавшие соответствующие стадии, разделились на частично перекрывавшиеся кластеры. Разделение на кластеры обеспечивалось первой главной компонентой РС1. Эта компонента связана с формой низкочастотных огибающих исходных сигналов, описывавших зависимости от времени энергии этих сигналов внутри временного блока. Таким образом, обработка с помощью метода главных компонент позволила кластеризовать акустико-эмиссионные сигналы по характеру их изменения со временем и установить связь между видом этих сигналов и соответствующей стадией деформационного упрочнения.

\section{4. Заключение}

В работе описано влияние стадийности процессов пластической деформации в алюминий-магниевом сплаве на регистрировавшуюся акустическую эмиссию. В качестве релевантных параметров, описывавших сигналы акустической эмиссии, были привлечены коэффициенты многоуровневых дискретных вейвлет-преобразований. Установлена связь этих параметров со стадиями деформационного упрочнения. Предложенный подход может быть использован при акустико-эмиссионном исследовании и диагностике деформационных процессов в алюминиевых сплавах.

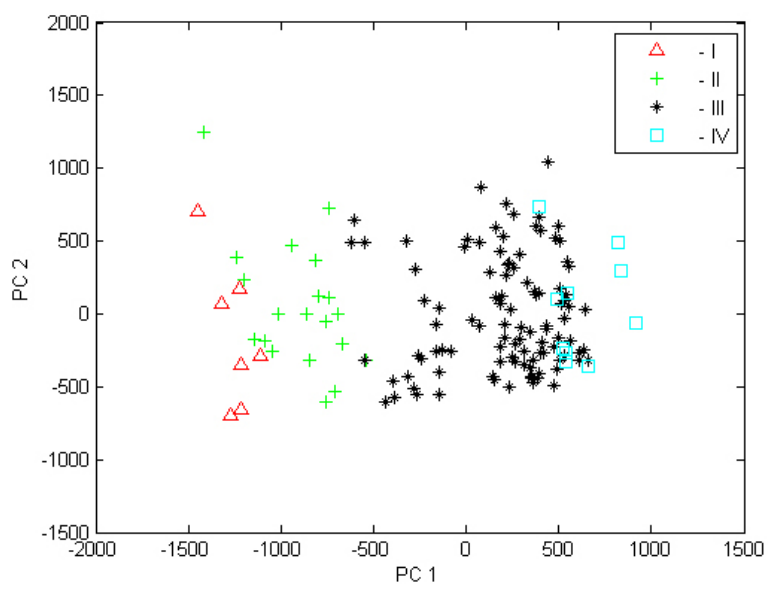

Рис. 2. Проекции характеристик сигналов акустической эмиссии на плоскость первых главных компонент.

Fig. 2. Projections frequency characteristics of acoustic emission signal on the plane of the first principal components. 
Благодарность/Acknowledgements. Статья подготовлена при финансовой поддержке РФФИ в рамках проекта №17-08-00914./The work was carried out at the financial support of the RFFI in the framework of the grant № 17-08-00914.

\section{Литература/References}

1. A. Marec, J.-H. Thomas, R Guerjouma. Mechanical Systems and Signal Processing. 22(6), 1441 - 1464 (2008). DOI: 10.1016/j.ymssp.2007.11.029

2. A. A. Lependin, V. V. Polykov. Technical Physics. 59(7), 1041 - 1045 (2014). DOI: 10.1134/S1063784214070184

3. D. B. B. Ferreira, R.R. Da Silva, J.M. A. Rebello, M.H. S. Siqueira. Insight. 46(5), $282-289$ (2004). DOI: 10.1784/insi.46.5.282.55560

4. S. A. Mallat. Wavelet Tour of Signal Processing. Academic Press. P. 795 (2009).

5. M.A. Hamstad, A. O'Gallagher, J. J Gary. Acoustic Emission. 20, 39-61 (2002).

6. N. Qing-Qing, I. Masaharu. Engineering Fracture Mechanics. 69(6), 717 - 728 (2002). DOI: 10.1016/S00137944(01)00105-9

7. A.V. Egorov, S. V. Kucheryavskiy, V.V. Polyakov. Chemometrics and Intelligent Laboratory Systems. 160, 8 - 12 (2017). DOI: 10.1016/j.chemolab.2016.11.007

8. K. H. Esbensen. Multivariate Data Analysis - In Practice. CAMO Process AS. P 160 (2002).

9. M. V. Markushev, M. Y. Murashkin. The Physics of Metals and Metallography. 92(1), 90-98 (2001). (in Russian) [М. В. Маркушев, М. Ю. Мурашкин. Физика металлов и металловедение. 92(1), 90 - 98 (2001).]

10. D. V. Mihlik, A.A. Shibkov. Izvestija Tulskogo gosudarstvennogo universiteta (in Russian) 3, 184-190 (2011). [Д. В. Михлик, А.А. Шибков. Известия Тульского государственного университета. Естественные науки. 3, 184 - 190 (2011)]

11. M. M. Krishtal, D.L. Merson. The Physics of Metals and Metallography. 81(1), 156-162 (1996). (in Russian)
[М.М. Криштал, Д.Л. Мерсон. Физика металлов и металловедение. 81(1), 156 - 162 (1996)]

12. S. A. Barannikova, V.I. Danilov, L.B. Zuev. Technical Physics. 74(10), 52-56 (2004). (in Russian) [С. А. Баранникова, В. И. Данилов, Л. Б. Зуев. Журнал технической физики. 70(10), 52 - 56 (2004)]

13. M.M. Krishtal. Physical Mesomechanics. 7(5), 5-29 (2004). (in Russian) [M. М. Криштал. Физическая мезомеханика. 7(5), 5-29 (2004).]

14. P. V. Trusov, E. A. Chechulina. Vestnik of Perm national research polytechnic university. Mechanics. 3(1) 186-232 (2014).(inRussian)[П. В.Трусов,Е. А.Чечулина.Вестник Пермского национального исследовательского политехнического университета. Механика. 3(1), 186-232 (2014).] DOI:10.15593/perm.mech/2014.3.10

15. A. V. Egorov, V. V. Polykov, E. A. Gumirov, A. A. Lependin. Instruments and Experimental Techniques. 48(5), 667 - 670 (2005). DOI: 10.1007/s10786-005-0119-6

16. A. A. Shibkov, M. A. Zeltov, A.E. Zolotov, A. A. Denisov. Vestnik of Tambov state university. 15(3), 1269-1273 (2010). (in Russian) [А.А. Шибков, М.А. Желтов, A.Е. Золотов, А.А. Денисов. Вестник Тамбовского государственного университета. 15(3), 1269-1273 (2010).]

17. S. V. Makarov, V. A. Plotnikov, E. A. Kolubaev. Izvestiya of Altai state university. 81(2), $207-210$ (2014). (in Russian) [C.В. Макаров, В.А. Плотников, Е.А. Колубаев. Известия Алтайского государственного университета. 81(2), $207-210$ (2014).] DOI: 10.14258/ izvasu(2015)1.2-05

18. V.V. Gorbatenko, V.I. Danilov, L.B. Zuev. Technical Physics. 87(3), 372-377 (2004). (in Russian) [В.В. Горбатенко, В.И. Данилов, Л.Б. Зуев. Журнал технической физики. 87(3), $372-377$ (2017).] DOI: 10.21883/JTF.2017.03.44241.1818

19. L. Yang, Y.C. Zhou, W.G. Mao, C.Lu. Applied Physics Letters. 93(23), 1 - 3 (2008). DOI: 10.1063/1.3043458

20. E. Hamdi, A. Le Duff, S. Laurent. Applied Acoustics. 74(5), 746 - 757 (2013). DOI: 10.1016/j.apacoust.2012.11.018 